\title{
An impedimetric immunosensor based on magnetic graphene nanoribbons modified by boronic acid for sensitive label-free detection of lymphoma cancer cells
}

\author{
H. Bagheri' ${ }^{\text {P. Hashemi }}{ }^{2}$, A. Afkhami² \\ ${ }^{1}$ Chemical Injuries Research Center, Baqiyatallah University of Medical Sciences, Tehran, Iran, \\ 2 Faculty of Chemistry, Bu-Ali Sina University, Hamedan, Iran \\ pegi.hashemi@gmail.com
}

\begin{abstract}
:
Cancer, as the second most common cause of death in the world, present a major and unmet challenge to healthcare globally. It has been reported that very sensitive monitoring of cancer cells could provide an easier and more effective way to monitor the progression of the disease. Thus, there is an immediate need for new detection and identification methods. The integral membrane protein CD20 was first identified as a marker for B cells over 20 years ago [4]. In this work, for label-free and direct detection of non-Hodgkin's lymphoma (NHL) in early stage, an impedimetric immunosensor based on a magnetic graphene nanoribbons (MGNRs) modified by boronic acid, anti-body and BSA (MGNRs/BA/Ab/BSA) composite was fabricated and evaluated. The physicochemical and analytical measurements were performed to examine the properties of nanocomposite and to determine the influence upon immunosensor performance to CD20 antigen detection. Impedimetric measurements showed significant changes in charge transfer resistance upon binding of cancer cells. The measurements were highly target specific, linear with logarithmic cell concentrations in PBS and human blood sample across a 100 cells $/ \mathrm{mL}$ and 1000000 cells $/ \mathrm{mL}$ range and associated with a detection limit of 38 cells $/ \mathrm{mL}$.
\end{abstract}

Key words: early detection of non-Hodgkin's lymphoma cancer, CD20 biomarker, B- cell lymphomas, immpedimetric immunosensor, graphene nanoribbons

\section{Introduction}

Early and accurate diagnosis is essential for the effective and ultimately successful treatment of cancers [1]. CD20 is a B cell-specific antigens [2] that are expressed on mature B cells and in most B-cell non-Hodgkin's lymphomas but not on early B-cell progenitors or later mature plasma cells [3] whose nonglycosylated 33 to $37 \mathrm{kD}$ phosphoprotein expressed on greater than $90 \%$ of B- cell lymphomas. The integral membrane protein CD20 was first identified as a marker for B cells over 20 years ago CD20 has been regarded as an attractive target for antibody therapy for the treatment of nonHodgkin's lymphoma (NHL) [5].

The most recent efforts in cancer cell detection have focused on biosensors with good sensitivity and selectivity, as well as rapid and easy operation [1].

Electrochemical affinity biosensors have been attractive for a broad range of applications in clinical diagnosis, biomedical research and environmental monitoring Because of their simplicity, rapid response, and compatibility with miniaturization. In particular, electrochemical immunosensors, relying on the specific antigen-antibody interaction, are the most widely used thanks to some of their peculiar features [6].

Here, we take advantage of the Rituximab monoclonal anti-CD20 antibody which specifically binds to the $B$ cells membrane surface, to introduce a novel strategy for specific detection of lymphomas cancer cells. For this propose we developed a new label free immpedimetric immunosensor. Synthesized Magnetic graphene nanoribbons (MGNRs) were modified by boronic acid (BA) and antibody (Ab) immobilized on the MGNRs/BA. After interaction of MGNRs/BA/Ab by cancer cell, the modified nanocomposite were magnetically collected on the SPCE where placed in a new home-made electrochemical cell and impedance signal was recorded. Affective variables on the immunosensor operation were 
studied and optimized. Different modification steps of nanocomposite were characterized by various techniques such as Resonance light scattering (RLS), fluorescence, electrochemical impedance spectroscopy (EIS) and cyclic voltammetry (CV).

\section{Results and discussion}

EIS is an electrochemical technique based on analysis of the linear response of the perturbation/response ratio when a low amplitude sinusoidal perturbation is applied. It is a powerful tool for measurement of faradaic processes involve charge transfer between the electrode and some species to be oxidized or reduced in the solution [7].

EIS and CV methods were used to characterization of various fabrication steps of the proposed impedimetric immunosensor. Fig. 1 illustrates the obtained Nyquist plots (fig. 1a) and cyclic voltammograms (fig. 1b) from various modification steps of the different impedimetric layer. The obtained results indicated that immunosensor was successfully prepared.
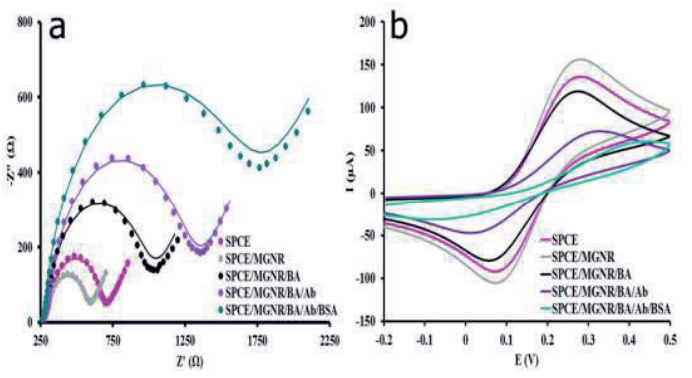

Fig. 1 a) Nyquist plots and b) cyclic voltammograms at different modification steps of the MGNR surface for fabrication of the immunosensor.

Under the optimized conditions the electrochemical performance of the proposed biosensor for cancer cell assay was investigated by impedance method and the EIS responses against the logarithm of different concentration of cells were shown in Fig. 2. It can be observed that the Rct increases with the increase of cancer cells concentration. Change in Rct parameters were investigated and calculated by the eq. (1) [8]:

$\Delta R_{C T}=R_{C T \text { (cancer cell) }}-R_{C T \text { (BSA) }}(1)$

Where $R_{C T \text { (cancer cell) is value of the resistance }}$ when cancer cells incubated with immobilized antibody and $R_{\mathrm{CT}}(\mathrm{BSA})$ is the resistance of proposed immunosensor after blocking by BSA.

To evaluate the analytical applicability of the developed method, human blood samples were evaluated as the complex systems model and cancer cells were analyzed as one type of circulating tumor cells (CTCs) in blood samples. Obtained satisfied results indicating that the proposed immunosensor may be a promising approach for B lymphocyte of lymphoma assay in blood sample without the significant matrix effect of the real sample.

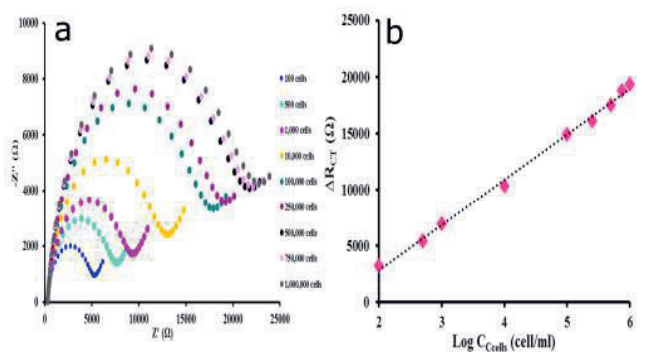

Fig. 2. immunosensor Nyquist plots incubated with different cancer cell concentration and impedimetric immunosensor Calibration curve for cancer cell detection ( $\triangle R C T$ vs $\log C)$.

\section{References}

[1]. C.Ding, Y.Ge, S. Zhang, Electrochemical and electrochemiluminescence determination of cancer cells based on aptamers and magnetic beads. Chemistry-A European Journal 16, 10707 10714 (2010); doi: 10.1002/chem.201001173.

[2]. N. A. Johnson, M. Boyle, A. Bashashati, S. Leach, A. Brooks-Wilson, L. H. Sehn, M. Chhanabhai, R. R. Brinkman, J. M. Connors, A. P. Weng, Diffuse large B-cell lymphoma: reduced CD20 expression is associated with an inferior survival. Blood 113, 3773-3780 (2009); doi: 10.1182/blood-2008-09-177469.

[3]. D. G. Maloney, Anti-CD20 antibody therapy for Bcell lymphomas. New England Journal of Medicine, 366, 2008-2016 (2012); doi: 10.1056/NEJMct1114348.

[4]. J. A. Ernst, H. Li, H. S. Kim, G. R. Nakamura, D. G. Yansura, R. L. Vandlen, Isolation and characterization of the B-cell marker CD20. Biochemistry, 44, 15150-15158 (2005); doi: 10.1021/bi0511078.

[5]. L. Tan, P. Lin, M. M. Chisti, A. Rehman, X. Zeng, Real time analysis of binding between rituximab (anti-cd20 antibody) and b lymphoma cells. Analytical chemistry, 85, 8543-8551 (2013); doi: 10.1021/ac400062v.

[6]. N. Xia, X. Wang, J. Yu, Y. Wu, S. Cheng, Y. Xing, L. Liu, Design of electrochemical biosensors with peptide probes as the receptors of targets and the inducers of gold nanoparticles assembly on electrode surface. Sensors and Actuators B: Chemical, 239, 834-840 (2017); doi: 10.1016/j.snb.2016.08.079.

[7]. F. S. Felix, L. Angnes, Electrochemical immunosensors-A powerful tool for analytical applications. Biosensors and Bioelectronics, 102, 470-478 (2017); doi: 10.1016/j.bios.2017.11.029.

[8]. A. Afkhami, P. Hashemi, H. Bagheri, J. Salimian, A. Ahmadi, T. Madrakian, Impedimetric immunosensor for the label-free and direct detection of botulinum neurotoxin serotype $A$ using $\mathrm{Au}$ nanoparticles/graphene-chitosan composite. Biosensors and Bioelectronics, 93, 124-131 (2017); doi: 10.1016/j.bios.2016.09.059. 